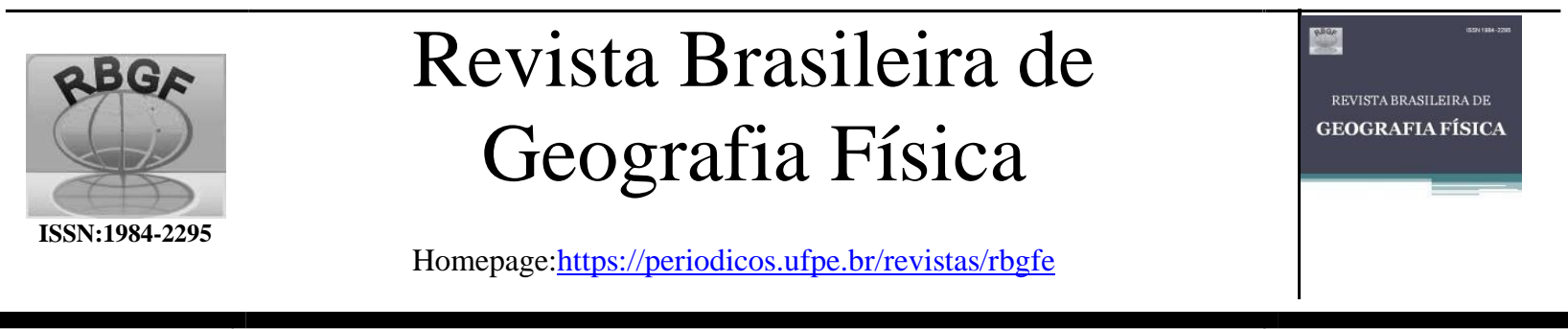

\title{
Avaliação da Acurácia Vertical dos Modelos Digitais de Elevação SRTM, ALOS World 3D e ASTER GDEM: Um Estudo de Caso no Vale dos Vinhedos, RS - Brasil
}

\author{
Jorge Antônio Viel ${ }^{1}$, Kátia Kellem da Rosa² ${ }^{2}$ Cláudio Wilson Mendes Junior ${ }^{2}$
}

\begin{abstract}
${ }^{1}$ Assistente em Administração do Instituto Federal do Rio Grande do Sul (IFRS), Campus Sertão. Mestre em Sensoriamento Remoto na UFRGS. Rodovia RS-135, KM 25 - Distrito Eng. Luiz, Estr. p/ Englert, Sertão - RS, 99170-000, e-mail: ja-viel89@ @otmail.com (Autor Correspondente). ${ }^{2}$ Professores, Doutores. Programa de Pós-Graduação em Sensoriamento Remoto / UFRGS. Avenida Bento Gonçalves, 9500 - Agronomia, Porto Alegre - RS, 91509-900, e-mail: katiakellem@gmail.com; geoclaudio@yahoo.com.br.
\end{abstract}

Artigo submetido em 22/12/2019 e aceite em 04/05/2020

\section{R E S U M O}

Este estudo tem como objetivo avaliar a acurácia vertical dos Modelos Digitais de Elevação (MDEs) SRTM v.3, ALOS World 3D e ASTER GDEM v.2 na região da denominação de origem Vale dos Vinhedos, RS. Para tanto, os dados desses MDEs, com resolução espacial de $30 \mathrm{~m}$, foram comparados com os de um MDE fotogramétrico com resolução espacial de $5 \mathrm{~m}$ no terreno, por meio de análises de regressão e correlação linear, e de perfis topográficos derivados desses modelos. O Padrão de Exatidão Cartográfica dos Produtos Cartográficos Digitais (PEC-PCD) de cada MDE foi analisado, para identificar a escala máxima de seu uso em estudos morfométricos, nas escalas 1:25.000, 1:50.000 e 1:100.000, por meio de cálculos da Tolerância Vertical e do Erro Médio Quadrático (EMQ). Os MDEs SRTM v.3 e ASTER GDEM v.2 atenderam o PEC-PCD altimétrico classe A na escala 1:100.000. Diferentemente do MDE ALOS World 3D que se enquadrou na classe B para a escala de 1:100.000. Todos os modelos, na escala 1:50.000, enquadraram-se na classe D, enquanto na escala 1:25.000 não houve enquadramento. O MDE SRTM v.3 foi o que apresentou melhores resultados morfométricos e o maior coeficiente de correlação de Pearson ( $\mathrm{r}=0,995)$. Todos os MDEs avaliados neste estudo apresentaram morfologia próxima a do MDE fotogramétrico. Portanto, recomenda-se o uso de todos os MDEs analisados em estudos morfométricos da área de estudo, sendo necessário observar o objetivo do trabalho, bem como a escala de análise e a apresentação desses dados.

Palavras-chave: Acurácia Vertical, SRTM v.3, Alos World 3D, ASTER GDEM v.2.

\section{Evaluation of the Vertical Acuracy of Digital Elevation Models SRTM, ALOS WORLD 3D and ASTER GDEM: a case study in Vale dos Vinhedos, RS - Brazil}

\begin{abstract}
A B S T R A C T
This work aims to evaluate the vertical accuracy of the digital elevation models (DEMs) SRTM v.3, Alos World 3D and ASTER GDEM v.2 in Vale dos Vinhedos designation of origin (DO) region, RS. Thus, the DEM data with $30 \mathrm{~m}$ of the spatial resolution were compared with photogrammetric DEM data with $5 \mathrm{~m}$ of the spatial resolution by linear regression and correlation analyzes, and also, topographic profiles carried out with these models. The Cartographic Accuracy Standard (PEC) of each DEM was analyzed to identify the maximum scale for morphometric application, in scales 1:25.000, 1:50.000 and 1:100.000, by calculations of Vertical Tolerance and the Mean Square Error (MSE). All DEMs. All the models studied were classified in class A for the 1:100,000 scale, and for the 1:50,000 scale the analyzed models were classified in class C, while in 1:25.000 scale doesn't have application. The DEM SRTM v.3 presented smaller altimetry errors compared to ASTER GDEM and Alos World 3D, as for mophometric analysis and Pearson correlation coefficient $(\mathrm{r}=0,995)$. It is worth mentioning that all models analyzed are statistically and morphologically close. Therefore, they can be used to conduct several studies, however it is necessary to have in mind the goal of the work, and the scale of analysis and presentation.
\end{abstract}

Keywords: Vertical accuracy, SRTM v.3, Alos World 3D, ASTER GDEM 


\section{Introdução}

A evolução das geotecnologias permitiu o aprimoramento e a produção de diversos mapeamentos. Dentre as geotecnologias, cabe destacar o Sensoriamento Remoto, o Geoprocessamento e os Sistemas de Posicionamento Global (GPS). O avanço da capacidade de processamento de dados permitiu a análise e manipulação de milhares de informações. $\mathrm{Na}$ área do Geoprocessamento tornou-se factível a integração entre diversos planos de informação, sendo possível realizar as mais variadas análises topológicas. Os sistemas de posicionamento global, por meio de pares de coordenadas, permitem a localização de objetos na superfície terrestre com precisão de milímetros. Por meio do Sensoriamento Remoto pode-se imagear toda a superfície terrestre e identificar objetos de vários tamanhos, desde oceanos até objetos medindo poucos centímetros.

Dentre as mais variadas evoluções na área de Sensoriamento Remoto, destaca-se a criação de Modelos Digitais de Elevação (MDEs). Esses modelos são "[...] arquivos que contêm registros altimétricos estruturados em linhas e colunas georreferenciadas, como uma imagem com um valor de elevação em cada pixel [...]" (Valeriano, p. 21, 2008). Diversas análises podem ser realizadas por meio dos MDEs. Valeriano (2008) ressalta que, embora a variável topográfica seja importante, diversos outros dados podem ser extraídos dos MDEs, como declividade, curvatura vertical e horizontal, direção de fluxo entre outros.

Para que os resultados obtidos por meio da utilização de MDEs sejam confiáveis, são necessários uma série de estudos que possibilitem a tomada de decisão do usuário. Sendo assim, autores como Lousada e Fernandes (2017), afirmam que é necessário saber até que ponto estes MDE são precisos para serem utilizados em estudos. Desta forma um parâmetro que deve ser verificado é a acurácia vertical.

Diversos estudos já foram realizados utilizando esses modelos, como exemplos, citamse os trabalhos desenvolvidos por Steink e Sano (2011), no qual os autores utilizaram índices morfométricos obtidos por meio de MDE, para a compartimentação geomorfológica semiautomatizada do Distrito Federal, Brasil. Gaida et al. (2016), avaliaram a influência do relevo na resposta espectral da vegetação, para tal, os autores utilizaram o MDE ASTER GDEM (Advanced Spaceborne Thermal Emission and Reflection Radiometer - Global Digital Elevation
Map). Os MDEs são utilizados em estudos que buscam a caracterização física de diversos locais, destes, podem-se incluir áreas de grandes extensões como em estudo realizado por Robaina et al. (2010), que utilizaram o MDE SRTM como auxílio à compartimentação geomorfológica da bacia hidrográfica do Ibicuí, localizada no estado do Rio Grande do Sul. Autores como Hoff et al. (2015), utilizaram o MDE SRTM (Shuttle Radar Topography Mission) como auxílio na caracterização geológica e geomorfológica de indicações geográficas para vinhos de qualidade.

Vários autores analisaram a acurácia vertical de diversos MDEs. Os modelos SRTM e ASTER GDEM são analisados frequentemente. Como exemplo, pode-se citar Souza (2015), que avaliou a acurácia vertical dos MDEs Topodata, ASTER GDEM e SRTM. Na análise realizada por esse autor, o MDE ASTER GDEM foi o que obteve melhor resultado, porém, deve-se destacar que a análise varia em função das peculiaridades da área de estudo. Araújo et al. (2019), analisaram a acurácia vertical de MDEs para a bacia hidrográfica do rio Piranha-Açu no Rio Grande do Norte. Da mesma forma, Girão et al. (2019), analisaram a acurácia vertical de MDEs para o estado do Rio de Janeiro.

Atualmente existem outros modelos disponibilizados gratuitamente, porém, com poucos estudos sobre os mesmos. Como exemplo, pode-se citar o MDE ALOS World 3D, gerado a partir do sensor PRISM (Panchromatic Remotesensing Instrument for Stereo Mapping) a bordo do satélite ALOS (Advanced Land Observing Satellite), disponibilizado gratuitamente pela Japan Aerospace Exploration Agency (JAXA).

Sendo assim, o objetivo deste trabalho foi avaliar a acurácia vertical dos MDEs ALOS World 3D, SRTM v.3 e ASTER GDEM v.2 na região da denominação de origem (DO) para vinhos de qualidade Vale dos Vinhedos, utilizando como verdade terrestre um MDE gerado a partir de levantamento aerofotogramétrico. Pois se acredita que a escolha do MDE é um fator importante para o desenvolvimento de análises que envolvam variáveis topográficas. Influenciando diretamente no resultado do trabalho.

A análise da acurácia vertical de diferentes MDEs disponíveis para a área de estudo e região do entorno foi necessária, pois possibilitou determinar para quais mapeamentos e escalas os mesmos poderiam ser utilizados. Desta forma, os resultados podem ser utilizados como base para a escolha de 
MDEs na aplicação de estudos em outras áreas que não possuam um MDE de maior acurácia vertical.

\section{Material e métodos}

\section{Localização da área de estudo}

A região da DO para vinhos de qualidade Vale dos Vinhedos está localizada na região nordeste do estado Rio Grande do Sul, abrangendo parte dos municípios de Bento Gonçalves, Garibaldi e Monte Belo do Sul (Figura 1). A área geográfica da denominação de origem para vinhos de qualidade tem $72,45 \mathrm{~km}^{2}$ (TONIETTO et al., 2013)

Essa região, conhecida como "Serra Gaúcha", foi colonizada no final do século XIX, principalmente por imigrantes italianos, e tem no setor vitivinícola uma importante fonte de renda. O ecoturismo e o turismo enológico estão em amplo desenvolvimento. Segundo Falcade (2011, p. 25 26) "no nordeste do Rio Grande do Sul, na região da Serra Gaúcha, a vitivinicultura teve seu maior crescimento, com importância econômica para milhares de produtores".

A área de estudo está inserida na Bacia Hidrográfica Taquari-Antas. A mesma está localizada no Bioma Mata Atlântica, existindo fragmentos de vegetação preservados devido à declividade de algumas áreas, impossibilitando práticas agrícolas. Segundo o Ministério do Meio Ambiente (MMA, 2004), a cobertura vegetal nas vertentes é composta pela Floresta Estacional Decidual e nos topos dos morros pela Floresta Ombrófila Mista, mas atualmente a vegetação apresenta-se degradada, principalmente no topo dos morros, pois geralmente são áreas com menores declividades, mais adequadas às práticas agrícolas. De acordo com a classificação climática de Koppen essa região enquadra-se em um clima $\mathrm{Cfb}$, caracterizado por apresentar estações do ano bem definidas com verão ameno e chuvas uniformemente distribuídas. Segundo dados da estação meteorológica localizada na Embrapa Uva e Vinho, no município de Bento Gonçalves, as normais climatológicas apresentam uma temperatura média anual de $17,8{ }^{\circ} \mathrm{C}$ e uma precipitação média anual de $1889 \mathrm{~mm}$.

A geologia da área de estudo pertence à Formação Serra Geral (CPRM, 2008), ocorrendo nas maiores altitudes rochas ácidas como riodacitos e riolitos (fácies Caxias), enquanto que nas menores altitudes encontram-se rochas básicas como os basaltos (fácies Gramado). A Serra Geral foi formada por uma sucessão de derrames oriundos de vulcanismo fissural, que ocorreram durante o período Cretáceo Inferior da era Mesozóica (248,2 a $65 \mathrm{Ma})$. A área de estudo possui como morfoestrutura a Bacia Sedimentar do Paraná e como morfoescultura o Planalto Meridional. A hidrografia é encaixada e desenvolveu-se sobre fraturas e falhas na crosta. $\mathrm{O}$ modelado possui vales encaixados e topos ondulados, com topografia diversificada, de relativo gradiente altimétrico, variando de 376 a $724 \mathrm{~m}$. As litologias mais resistentes aos processos de dissecação atuam como divisores de água Materiais

Para o desenvolvimento do trabalho foram utilizados quatro MDEs, mas apenas três passaram pela análise da acurácia vertical, pois utilizou-se o MDE gerado a partir de levantamento aerofotogramétrico como verdade do terreno. Sendo assim, realizou-se a análise da acurácia vertical dos MDEs SRTM v.3, ASTER GDEM v. 2 e ALOS World 3D.

O levantamento aerofotogramétrico foi realizado em 01 de novembro de 2005 pela empresa Multiespectral Mapas Digitais. A restituição fotogramétrica objetivou produtos para a escala de trabalho de 1:5000. Como produtos finais foram gerados um ortofotomosaico, com resolução espacial de $60 \mathrm{~cm}$ e curvas de nível de equidistância de 5 metros entre as mesmas e acurácia vertical de $50 \mathrm{~cm}$.

Para gerar o MDE realizou-se a interpolação das curvas de nível, disponibilizadas pela Embrapa Uva e Vinho, por meio de uma Rede Triangular Irregular (RTI). O método RTI foi adotado neste estudo, pois segundo Maune et al. (2007), este representa de maneira satisfatória regiões de relevo irregular, evidenciando descontinuidades na inclinação do terreno como picos, depressões, divisores de águas, vales e desfiladeiros. Converteu-se essa RTI em um MDE com resolução espacial de $5 \mathrm{~m}$, interpolado pelo método Vizinho Natural.

O MDE SRTM v.3 (Shuttle Radar Topography Mission) é um modelo criado pela National Aeronautics and Space Administration (NASA), em parceria com a National Geospatial Intelligence Agency (NGA). Segundo Rodrigues et al. (2006), esse projeto realizou mapeamento topográfico da superfície terrestre, a partir de dois radares acoplados ao ônibus espacial Endeavour. Foram obtidas imagens desses sensores em latitudes menores que $60^{\circ}$, que foram utilizadas para a geração de imagens interferométricas da 
superfície terrestre e de MDEs com resolução espacial de $30 \mathrm{~m}$. Conforme esses autores, para a
América do Sul, $90 \%$ dos pontos analisados apresentaram um erro altimétrico inferior a $9 \mathrm{~m}$..

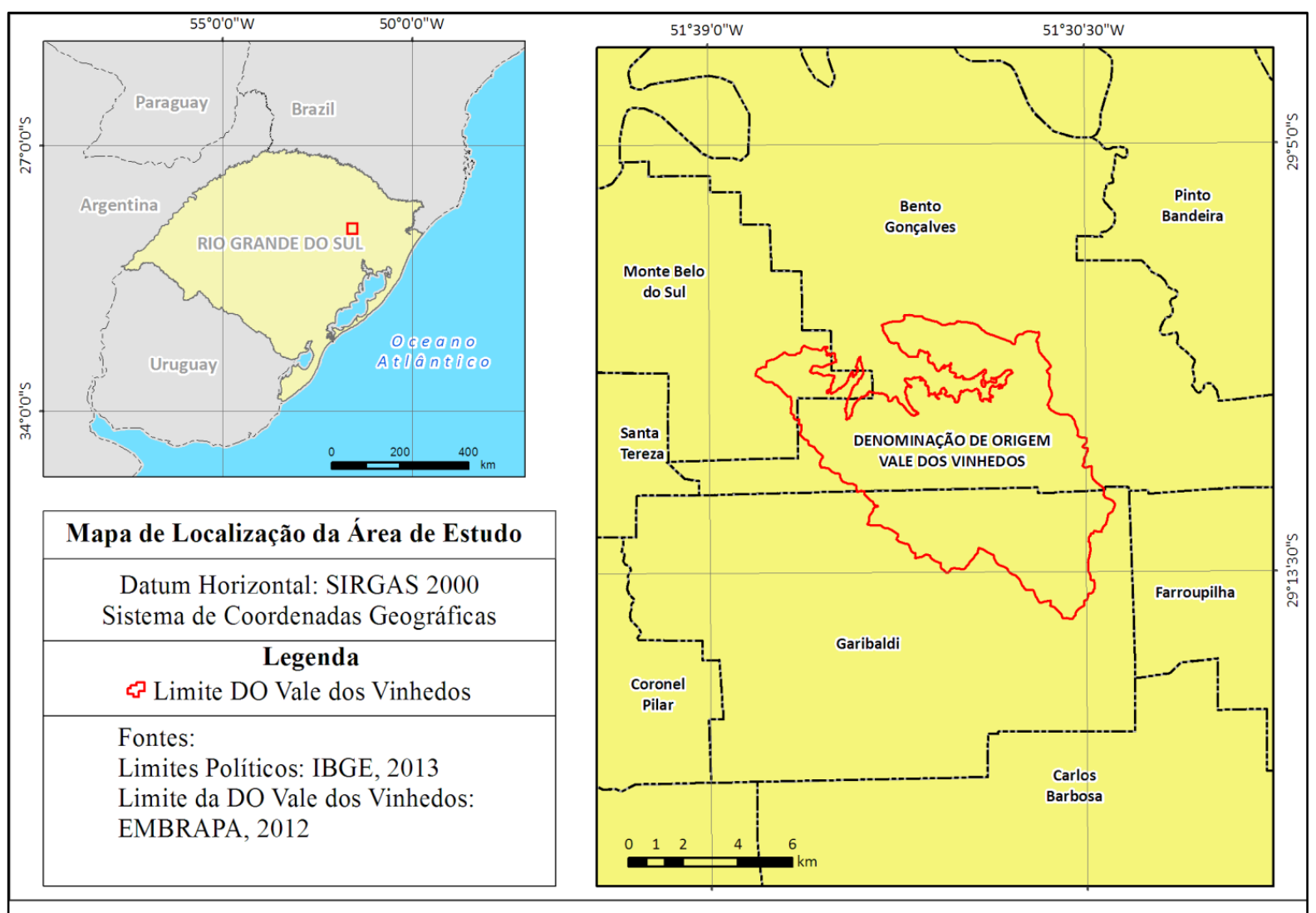

Figura 1. Mapa de localização. Área de abrangência da DO Vale dos Vinhedos inserida em parte dos municípios de Bento Gonçalves, Garibaldi e Monte Belo do Sul.

O MDE ALOS World 3D foi gerado a partir do sensor PRISM (Panchromatic Remotesensing Instrument for Stereo Mapping) a bordo do satélite ALOS (Advanced Land Observing Satellite), foi gerado pela Japan Aerospace Exploration Agency (JAXA). Essa missão teve a duração de 9 anos, iniciando em 2005 e terminando em 2014, sendo seus dados disponibilizados no ano de 2014. Segundo Tadono et al. (2014), esse modelo possui resolução espacial de 30 metros e acurácia vertical de 5 metros. Segundo os mesmos autores o PRISM era um sensor óptico com três radiômetros, tendo, um deles a visada nadir, outro para frente (forward) e outro para trás (backward).

Outro modelo utilizado no trabalho foi o ASTER GDEM v.2, o modelo foi gerado em cooperação entre a NASA e o Ministério da Economia, Industria e Trabalho Japonês. O MDE ASTER GDEM v.2 foi lançado em 2011. O radiômetro de emissão e reflexão térmica espacial (ASTER) é um sensor a bordo do satélite Terra que usa câmeras no comprimento infravermelho, tendo duas visadas uma no nadir e a outra backnadir. Segundo Tachikawa et al. (2011), esse modelo possui resolução espacial de 30 metros e acurácia vertical que varia dependendo da área. Segundo os mesmos autores, esse modelo possui acurácia vertical de 6,1 metros em áreas planas e abertas, já para áreas de relevo irregular e florestadas esse modelo possui acurácia vertical de 15,1 metros.

\section{Métodos}

O teste da acurácia vertical dos MDEs para a área da DO Vale dos Vinhedos foi desenvolvido por meio de técnicas estatísticas que permitiram analisar as estatísticas básicas dos MDEs como média e desvio padrão, da mesma forma, foram analisadas as estatísticas das classes dos produtos morfométricos derivados dos MDEs, como a declividade. Também foram analisados os perfis topográficos para comparação entre os modelados da superfície. Essa tarefa foi realizada para demonstrar o MDE que melhor representa a morfologia da região, tendo como base o perfil topográfico do MDE gerado a partir dos dados do 
aerolevantamento. Foram gerados dois segmentos (A-B) e (C-D), com extensão de 2.500 metros. Os mesmos têm sentido oeste/leste e rumo nordeste, sendo o valor da elevação coletado a cada 30 metros.

\section{Análises estatísticas}

A correlação entre os modelos foi determinada com base no coeficiente de correlação linear de Pearson. Segundo Feijoo (2010) esse coeficiente é baseado em modelos de regressão linear e busca determinar o nível de associação entre as amostras. Esse coeficiente varia de +1 à 1 , sendo +1 a correlação positiva perfeita e -1 a correlação negativa perfeita.

A declividade é uma variável importante para o entendimento da ocupação espacial, bem como dos processos geomorfológicos. Sendo assim, analisaram-se as declividades da área de estudo. Para a divisão da declividade em classes, utilizou-se o sistema adotado pela Embrapa (1979), conforme a Tabela 1.

Tabela 1. Distribuição das classes de declividade conforme Embrapa.

\begin{tabular}{cc}
\hline Declividade & Classe \\
\hline $0-3 \%$ & Plano \\
$3-8 \%$ & Suave Ondulado \\
$8-20 \%$ & Ondulado \\
$20-45 \%$ & Forte Ondulado \\
$45-75 \%$ & Montanhoso \\
$>75 \%$ & Escarpado \\
\hline
\end{tabular}

Para aprimorar a análise estatística dos dados de elevação dos MDEs, foi calculado o Erro Médio Quadrático (EMQ), apresentado na Equação 1. Esse teste avalia a qualidade do ajuste de um modelo e ele é obtido por meio da raiz da média do quadrado da diferença entre o que é testado e o valor real.

$$
\begin{aligned}
\mathbf{E M Q} & =\sqrt{\frac{\sum\left(\mathbf{Z}_{\mathbf{i}}-\mathbf{Z}_{\mathrm{t}}\right)^{2}}{\mathbf{n}-\mathbf{1}}} \\
& \text { Onde: } \\
& -\mathrm{Zi}=\mathrm{o} \text { valor altimétrico do MDE; } \\
& -\mathrm{Zt}=\mathrm{o} \text { valor do ponto de controle; } \\
& -\mathrm{n}=\text { número total de pontos de controle. }
\end{aligned}
$$

\section{Análises do padrão de exatidão cartográfica}

Realizou-se uma análise do Padrão de Exatidão Cartográfica dos Produtos Cartográficos Digitais (PEC-PCD) altimétrico dos MDEs. O PEC foi estabelecido, para produtos impressos, pelo Decreto 89.817, de 20 de Julho de 1984, que estabeleceu normas para a produção e representação de dados cartográficos. Os valores definidos no referido Decreto foram complementados pelos valores apresentados na norma de Especificação Técnica de Produtos de Conjuntos de Dados Geoespaciais (ET-PCDG, 2016), dividindo os produtos cartográficos em classes A, B, C ou D, sendo a Classe A um produto de melhor qualidade, as classes $\mathrm{B}$ e $\mathrm{C}$ um produto intermediário e a Classe $\mathrm{D}$ um produto de menor qualidade. Neste trabalho foram avaliados os dados correspondentes à elevação (PEC-PCD altimétrico), não considerando o PEC-PCD em relação ao erro horizontal (planimétrico).

O PEC-PCD foi analisado para as escalas 1:25.000, $1: 50.000 \quad$ e $1: 100.000$. Convencionalmente costuma-se apresentar curvas de nível com equidistância de $10 \mathrm{~m}$ para a escalas 1:25.000, de $20 \mathrm{~m}$ para escala $1: 50.000$ e de $50 \mathrm{~m}$ para escala 1:100.000, conforme trabalhos realizados por Santos et al. (2010), Dias et al. (2011) e Souza (2015).

Segundo o Decreto 89.817 de 20 de Julho de 1984 e a norma ET-PCDG de 2016, a tolerância vertical e o erro médio quadrático (EMQ) respeitam a equidistância entre as curvas de nível. A tolerância vertical para a Classe A deve conter um erro planimétrico inferior a 2,7 metros ou inferior a $1 / 6$ para o EMQ.. Para a Classe B deve ser de $1 / 2$ da equidistância entre as curvas de nível; já o EMQ deve ser $1 / 3$ da equidistância entre as curvas de nível. Para a classe $\mathrm{C}$, a tolerância vertical deve respeitar $3 / 5$ da equidistância entre as curvas de nível e o EMQ de 2/5 da equidistância entre as mesmas. Para a classe $\mathrm{D}$, a tolerância vertical deve respeitar $3 / 4$ da equidistância entre as curvas de nível e o EMQ de $1 / 2$ da equidistância entre estas. Para a obtenção da tolerância vertical foram analisados 1312 pontos dos MDEs; já para a análise do EMQ foram utilizados todos os pixels dos modelos. Na Tabela 2, pode-se observar os limites de tolerância vertical e EMQ para as escalas analisadas.

\section{Resultados e discussão}

Analisando os dados estatísticos dos MDEs (Tabela 3), observou-se que as médias dos MDEs ficaram próximas, sendo as médias dos MDEs SRTM v.3 e ASTER GDEM v.2 as que apresentaram maior proximidade com a média do MDE gerado dos dados fotogramétricos. Com 
relação aos dados de elevação, observou-se que o MDE SRTM v.3 registrou a elevação máxima mais aproximada do MDE fotogramétrico, com uma diferença de $9 \mathrm{~m}$. O MDE que apresentou maior diferença em relação à elevação máxima foi o ASTER GDEM v.2, com $17 \mathrm{~m}$ em relação ao MDE utilizado como verdade terrestre. Com relação à elevação mínima, o MDE que apresentou os resultados mais próximos aos valores do MDE fotogramétrico foi o ALOS World 3D, com diferença de $11 \mathrm{~m}$.

$\mathrm{Na}$ análise dos dados de declividade dos MDEs, ressalta-se a importância em se observar o erro inerente a cada modelo utilizado. Desta forma, ao utilizar esses dados é relevante considerar que a análise dos produtos inicia com um erro intrínseco, referente a cada modelo.

Tabela 2. Limites de tolerância vertical e erro médio quadrático para as escalas analisadas.

Fonte: ET-ADGV DEFESA F Ter (2016).

\begin{tabular}{|c|c|c|}
\hline Classe & $\begin{array}{c}\text { Tolerância } \\
\text { Vertical } \\
\text { (metros) }\end{array}$ & $\begin{array}{c}\text { Erro Médio } \\
\text { Quadrático } \\
\text { (metros) }\end{array}$ \\
\hline $\begin{array}{c}\text { Classe A de } \\
1: 25.000\end{array}$ & 2,70 & 1,67 \\
\hline $\begin{array}{c}\text { Classe B de } \\
1: 25.000\end{array}$ & 5,0 & 3,3 \\
\hline $\begin{array}{c}\text { Classe C de } \\
1: 25.000\end{array}$ & 6,0 & 4,0 \\
\hline $\begin{array}{c}\text { Classe D de } \\
1: 25.000\end{array}$ & 7,5 & 5,0 \\
\hline $\begin{array}{c}\text { Classe A de } \\
1: 50.000\end{array}$ & 5,50 & 3,33 \\
\hline $\begin{array}{c}\text { Classe B de } \\
1: 50.000\end{array}$ & 10,0 & 6,0 \\
\hline $\begin{array}{c}\text { Classe C de } \\
1: 50.000\end{array}$ & 12,0 & 8,0 \\
\hline $\begin{array}{c}\text { Classe D de } \\
1: 50.000\end{array}$ & 15,0 & 10,0 \\
\hline $\begin{array}{c}\text { Classe A de } \\
1: 100.000\end{array}$ & 13,70 & 8,33 \\
\hline $\begin{array}{c}\text { Classe B de } \\
1: 100.000\end{array}$ & 25,0 & 16,6 \\
\hline $\begin{array}{c}\text { Classe C de } \\
1: 100.000\end{array}$ & 30,0 & 20,0 \\
\hline $\begin{array}{c}\text { Classe D de } \\
1: 100.000\end{array}$ & 36,6 & 25,0 \\
\hline
\end{tabular}

Tabela 3. Dados estatísticos referentes à elevação dos MDEs analisados.

\begin{tabular}{lllll}
\hline Estatística & $\begin{array}{l}\text { Aerolev } \\
\text { antame } \\
\text { nto }\end{array}$ & $\begin{array}{l}\text { Wor } \\
\text { ld }\end{array}$ & $\begin{array}{l}\text { SRT } \\
\text { M }\end{array}$ & $\begin{array}{l}\text { ASTER } \\
\text { GDEM } \\
\text { v.2 }\end{array}$ \\
\hline $\begin{array}{l}\text { Elevação } \\
\text { máxima } \\
(\mathrm{m})\end{array}$ & 722 & 735 & 731 & 739 \\
$\begin{array}{l}\text { Elevação } \\
\text { mínima } \\
(\mathrm{m})\end{array}$ & 377 & 388 & 393 & 393 \\
$\begin{array}{l}\text { Elevação } \\
\text { média (m) }\end{array}$ & 570 & 576 & 575 & 575 \\
$\begin{array}{l}\text { Desvio } \\
\text { padrão } \\
(\mathrm{m})\end{array}$ & 65 & 64 & 64 & 64 \\
\hline
\end{tabular}

Analisando os dados de declividade (Tabela 4) dos MDEs para a área da DO Vale dos Vinhedos, concluiu-se que o MDE ALOS World 3D foi o modelo que teve a maior aproximação da declividade máxima obtida pelo MDE gerado pelo aerolevantamento. Com relação à declividade mínima, todos os três modelos apresentaram a mesma resposta, sendo essa muito próxima do MDE utilizado como verdade do terreno.

Tabela 4. Dados estatísticos referentes à declividade dos MDEs analisados.

\begin{tabular}{|c|c|c|c|c|}
\hline Estatísticas & $\begin{array}{l}\text { Aerolev } \\
\text { antamen } \\
\text { to }\end{array}$ & $\begin{array}{l}\text { Worl } \\
\text { d 3D }\end{array}$ & $\begin{array}{l}\text { SRT } \\
\text { M } \\
\text { v.3 }\end{array}$ & $\begin{array}{c}\text { ASTE } \\
\text { R } \\
\text { GDE } \\
\text { M v.2 }\end{array}$ \\
\hline $\begin{array}{l}\text { Declividad } \\
\text { e máxima } \\
(\%)\end{array}$ & 97,17 & $\begin{array}{c}101 \\
45\end{array}$ & $\begin{array}{c}88,9 \\
5\end{array}$ & 119,49 \\
\hline $\begin{array}{l}\text { Declividad } \\
\text { e mínima } \\
(\%)\end{array}$ & 0,031 & 0 & 0 & 0 \\
\hline $\begin{array}{l}\text { Declividad } \\
\text { e média } \\
(\%)\end{array}$ & 18,54 & $\begin{array}{c}20,5 \\
5\end{array}$ & $\begin{array}{c}18,4 \\
4\end{array}$ & 19,87 \\
\hline $\begin{array}{l}\text { Desvio } \\
\text { padrão }(\%)\end{array}$ & 11,32 & $\begin{array}{c}13,6 \\
4\end{array}$ & $\begin{array}{c}11,3 \\
1\end{array}$ & 12,34 \\
\hline
\end{tabular}

A divisão da declividade em classes permitiu identificar as diferenças entre os MDEs em relação à área ocupada por essa variável (Tabela 5). Com relação à classificação de relevo Plano, percebe-se que os MDEs obtiveram respostas semelhantes em relação à área de estudo. Porém quando analisa-se a área em hectares percebe-se uma diferença considerável entre o 
MDE gerado pelo aerolevantamento e o MDE ASTER GDEM v.2. O MDE ALOS World 3D teve maior similaridade com o modelo utilizado como verdade do terreno.

A classificação Suave Ondulado representou cerca de 1000 ha da área de estudo (Tabela 5), sendo o MDE SRTM v.3 o que obteve resultado mais aproximado do MDE gerado pelo aerolevantamento. $\mathrm{O}$ relevo Ondulado representa 43\% da área de estudo e novamente os dados gerados pelo MDE SRTM v.3 foram os que obtiveram resultado mais aproximado do MDE utilizado como verdade do terreno. O relevo Forte Ondulado é a segunda classe mais representativa, com $37 \%$ da região da denominação de origem Vale dos Vinhedos, sendo os dados de declividade a partir do MDE ALOS World 3D os que obtiveram maior similaridade.

O relevo escarpado possui a menor representatividade na área de estudo, ocupando em torno de 4 ha. Para esta classe, o modelo SRTM v.3 foi o que obteve maior proximidade em relação aos dados de declividade gerados pelo MDE fotogramétrico. Os resultados possibilitaram identificar que os MDEs ALOS World 3D e ASTER GDEM v.2 registraram para esta classe maior representatividade, ultrapassando $10 \mathrm{ha}$.

Tabela 5. Distribuição da declividade em relação à área de estudo para cada MDE analisado.

* Porcentagem em relação à área de estudo.

\begin{tabular}{lccccccccc}
\hline \multicolumn{1}{c}{ Classes } & \multicolumn{2}{c}{ Aerolevantamento } & \multicolumn{2}{c}{ World 3D } & \multicolumn{2}{c}{ SRTM v.3 } & \multicolumn{2}{c}{ ASTER GDEM } \\
& Área (ha) & $*$ & $\begin{array}{c}\text { Área } \\
\text { (ha) }\end{array}$ & $*$ & Área (ha) & $*$ & Área (ha) & $*$ \\
\hline $0-3 \%$ (Plano) & 225,81 & 3 & 221,13 & 3 & 240,75 & 3 & 173,34 & 2 \\
$3-8$ (Suave Ondulado) & 1059,30 & 14 & 981,45 & 14 & 1030,14 & 14 & 908,37 & 13 \\
$8-20 \%$ (Ondulado) & 3159,81 & 43 & 2853,00 & 39 & 3162,33 & 44 & 3016,08 & 42 \\
$20-45 \%$ (Forte & 2736,09 & 37 & 2743,38 & 38 & 2645,10 & 36 & 2844,81 & 39 \\
Ondulado) & 184,23 & 2,9 & 433,08 & 5,8 & 183,42 & 2,96 & 291,87 & 3,8 \\
$45-75 \%$ (Montanhoso) & 3,96 & 0,1 & 15,39 & 0,2 & 2,61 & 0,04 & 11,79 & 0,2 \\
$>75 \%$ (Escarpado) & & & & & & & & & \\
\hline
\end{tabular}

Desta forma, percebe-se que as declividades de maior representatividade são as situadas entre $8 \%$ e $45 \%$, englobando as classes de relevo Ondulado e Forte Ondulado respectivamente. Essas duas classes representam $80 \%$ da região da DO Vale dos Vinhedos. Cabe destacar que essa representatividade foi evidenciada pelos dados de declividades gerados para todos os MDEs avaliados. Na Figura 2 observa-se a distribuição espacial da declividade na área de estudo, derivada do MDE fotogramétrico. A análise estatística permitiu identificar uma forte correlação entre os valores dos MDEs testados com os do MDE fotogramétrico.

A correlação entre o MDE fotogramétrico e o ALOS World 3D pode ser observada na Figura 3. No gráfico é possível observar que existe uma forte correlação positiva entre esses dois MDEs. Quando analisada a elevação média, obteve-se diferença de $6 \mathrm{~m}$ entre os mesmos.
O coeficiente de correlação desses modelos obteve valor de $r=0,994$, indicando uma alta correlação linear entre esses MDEs. Os resultados encontrados são corroborados por diversos autores como Mello e Silveira (2018), que encontram resultados insatisfatórios para todos os modelos globais, porém o MDE que apresentou piores resultados foi o ASTER. Da mesma forma, Fran et al. (2017), também conseguiram melhores resultados para o MDE SRTM.

O MDE SRTM v.3 apresentou forte correlação com o modelo utilizado como verdade do terreno (Figura 4). O coeficiente de correlação apresentou valor de $r=0,995$, indicando assim uma alta correlação positiva entre esses dados. Analisando a média da elevação, observou-se uma diferença de $5 \mathrm{~m}$ entre os modelos. 


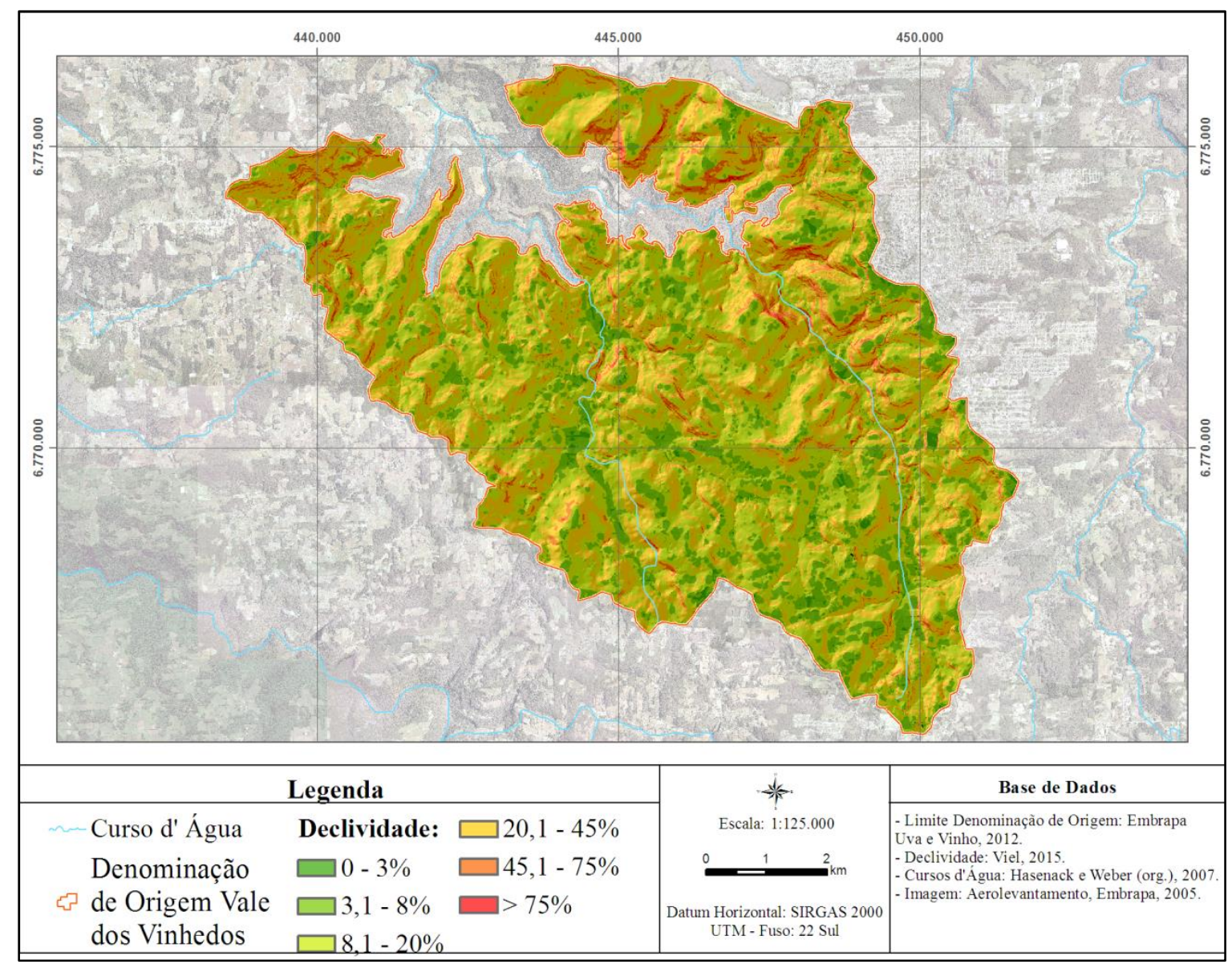

Figura 2. Mapa de declividade. Distribuição espacial da declividade na área de estudo.

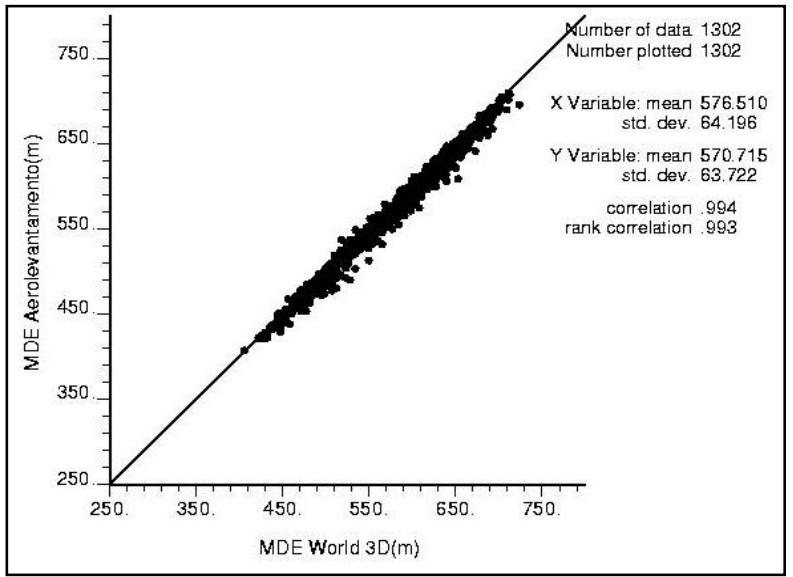

Figura 3. Correlação entre o MDE fotogramétrico e o MDE do ALOS World 3D.

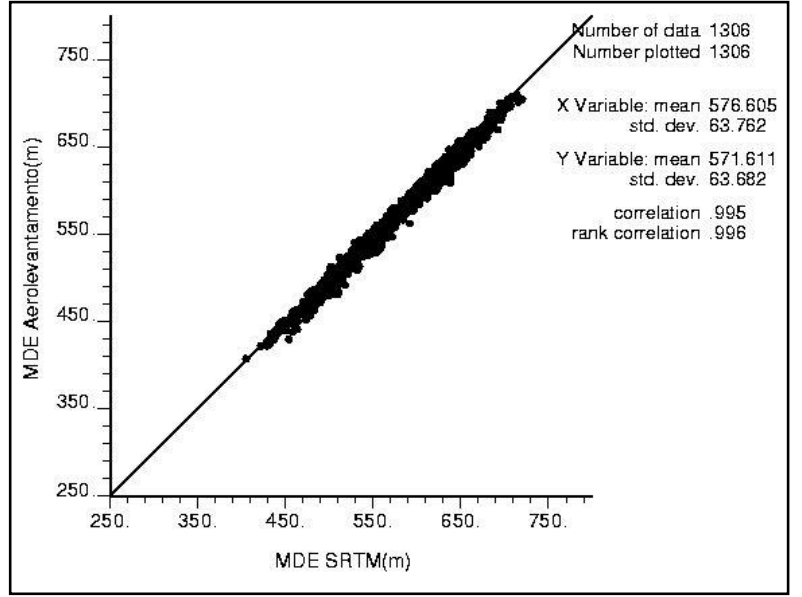

Figura 4. Correlação entre o MDE fotogramétrico e o MDE SRTM v.3.

A Figura 5 apresenta a correlação entre o MDEs ASTER GDEM v.2 e o gerado pelo aerolevantamento. A análise da correlação permite determinar uma forte correlação positiva entre esses dois MDEs. O valor do coeficiente de correlação obtido foi $r=0,994$, que indica uma

Viel, J. A., Rosa, K. K. Mendes Junior, C. W. 
forte correlação positiva. Os valores das médias da elevação apresentaram uma diferença de $5 \mathrm{~m}$ entre esses modelos.

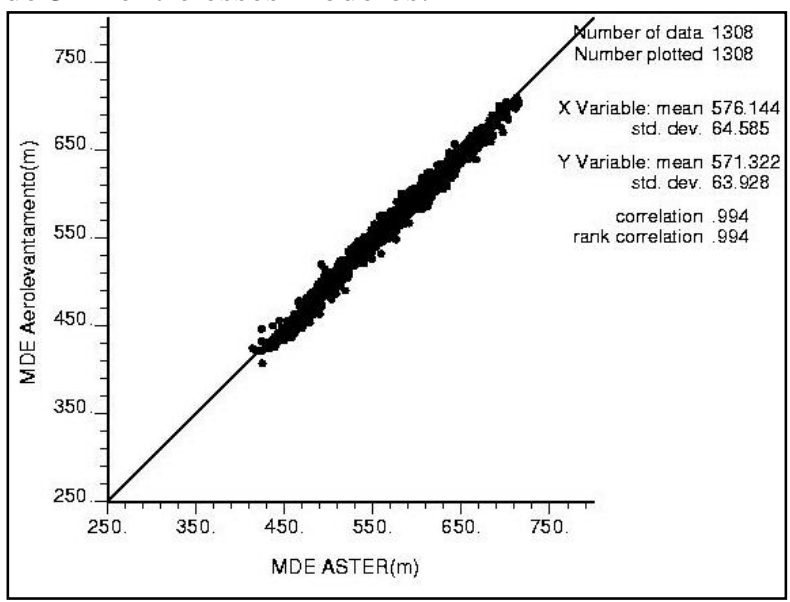

Figura 5. Correlação entre o MDE fotogramétrico e o MDE ASTER GDEM v.2.

Com a análise da correlação, observou-se que todos os MDEs testados apresentaram uma alta correlação com o MDE fotogramétrico, sendo que o melhor resultado foi obtido para o MDE SRTM v.3. Os resultados vão ao encontro de Morais et al. (2017), os quais ressaltam que a acurácia altimétrica depende do método de obtenção. $\mathrm{O}$ ASTER GDEM v.2 e ALOS World 3D são obtidos por estereoscopia de imagens ópticas, enquanto que o SRTM v.3 é gerado por interferometria de imagens de radar da banda $\mathrm{C}$.

A baixa acurácia da elevação do ASTER GDEM v.2 e do ALOS World 3D pode estar relacionada com a presença de nuvens, sombra, corpos d' água, como observado por Lang e Welch (1999).

O PEC-PCD permite classificar os produtos cartográficos conforme sua precisão. $\mathrm{Na}$
Tabela 6, estão descritas as análises estatísticas realizadas com os MDEs testados, para a classificação do PEC-PCD (altimétrico) de cada um destes dados.

Na região da DO Vale dos Vinhedos os modelos SRTM v.3 e ASTER GDEM enquadraram-se no PEC-PCD (altimétrico) classe A na escala de 1:100.000, tanto na tolerância vertical, quanto no EMQ. Fato que não ocorreu com o modelo ALOS World 3D que, para a escala de 1:100.000, enquadrou-se na classe B. Observando a tolerância vertical e o EMQ, observamos que todos os modelos se enquadraram na classe $\mathrm{D}$ para a escala 1:50.000. Os modelos não se enquadram nas classes A, B e C na escala 1:50.000 e nas classes A, B, C e D da escala 1:25.000. Por meio das análises realizadas constatou-se que o MDE SRTM foi o que obteve melhor resultado em relação ao PEC-PCD altimétrico. Valores semelhantes foram encontrados por Silva et al. (2018), para o MDE ALOS World 3D. Já o MDE SRTM v.3 Silva et al. (2018), enquadraram na classe B do PEC-PCD.

A análise dos perfis topográficos permitiu avaliar a morfologia apresentada pelos MDEs estudados. Na Figura 8, pode-se observar a localização dos segmentos utilizados para gerar os perfis topográficos. No segmento A/B (Figura 6) o perfil que apresentou maior semelhança morfológica com o MDE gerado pelo aerolevantamento foi o SRTM v.3. Por outro lado, o MDE que apresentou menor semelhança com o modelo fotogramétrico foi o ALOS World 3D, seguido pelo ASTER GDEM v2. 
Tabela 6. Análises estatísticas para o enquadramento do PEC-PCD(altimétrico).

\begin{tabular}{|c|c|c|c|}
\hline \multicolumn{4}{|c|}{ MDE ALOS World 3D } \\
\hline Escala & Classe & Tolerância Vertical ( $n^{\circ}$ de pontos) & $\begin{array}{l}\text { Erro Médio } \\
\text { Quadrático }\end{array}$ \\
\hline \multirow{4}{*}{$1: 25.000$} & A & $37 \%$ apresentaram elevação $\leq 2,7$ metros & \multirow{12}{*}{9,18 metros } \\
\hline & $\mathrm{B}$ & $53 \%$ apresentaram elevação $\leq 5$ metros & \\
\hline & $\mathrm{C}$ & $59 \%$ apresentaram elevação $\leq 6$ metros & \\
\hline & $\mathrm{D}$ & $67 \%$ apresentaram elevação $\leq 7,5$ metros & \\
\hline \multirow{4}{*}{$1: 50.000$} & A & $56 \%$ apresentaram elevação $\leq 5,5$ metros & \\
\hline & $\mathrm{B}$ & $77 \%$ apresentaram elevação $\leq 10$ metros & \\
\hline & $\mathrm{C}$ & $83 \%$ apresentaram elevação $\leq 12$ metros & \\
\hline & $\mathrm{D}$ & $90 \%$ apresentaram elevação $\leq 15$ metros & \\
\hline \multirow{4}{*}{$1: 100.000$} & A & $87 \%$ apresentaram elevação $\leq 13,7$ metros & \\
\hline & $\mathrm{B}$ & $98 \%$ apresentaram elevação $\leq 25$ metros & \\
\hline & $\mathrm{C}$ & $99 \%$ apresentaram elevação $\leq 30$ metros & \\
\hline & $\mathrm{D}$ & $100 \%$ apresentaram elevação $\leq 36,6$ metros & \\
\hline \multicolumn{4}{|c|}{ MDE SRTM v.3 } \\
\hline Escala & Classe & Tolerância Vertical ( $n^{\circ}$ de pontos) & $\begin{array}{l}\text { Erro Médio } \\
\text { Quadrático }\end{array}$ \\
\hline \multirow{4}{*}{$1: 25.000$} & A & $38 \%$ apresentaram elevação $\leq 2,7$ metros & \multirow{12}{*}{8,47 metros } \\
\hline & $\mathrm{B}$ & $57 \%$ apresentaram elevação $\leq 5$ metros & \\
\hline & $\mathrm{C}$ & $62 \%$ apresentaram elevação $\leq 6$ metros & \\
\hline & $\mathrm{D}$ & $71 \%$ apresentaram elevação $\leq 7,5$ metros & \\
\hline \multirow{4}{*}{$1: 50.000$} & A & $60 \%$ apresentaram elevação $\leq 5,5$ metros & \\
\hline & $\mathrm{B}$ & $82 \%$ apresentaram elevação $\leq 10$ metros & \\
\hline & $\mathrm{C}$ & $88 \%$ apresentaram elevação $\leq 12$ metros & \\
\hline & $\mathrm{D}$ & $93 \%$ apresentaram elevação $\leq 15$ metros & \\
\hline \multirow{4}{*}{$1: 100.000$} & A & $91 \%$ apresentaram elevação $\leq 13,7$ metros & \\
\hline & $\mathrm{B}$ & $99 \%$ apresentaram elevação $\leq 25$ metros & \\
\hline & $\mathrm{C}$ & $100 \%$ apresentaram elevação $\leq 30$ metros & \\
\hline & $\mathrm{D}$ & $100 \%$ apresentaram elevação $\leq 36,6$ metros & \\
\hline \multicolumn{4}{|c|}{ MDE ASTER GDEM v.2 } \\
\hline Escala & Classe & Tolerância Vertical ( $n^{\circ}$ de pontos) & $\begin{array}{l}\text { Erro Médio } \\
\text { Quadrático }\end{array}$ \\
\hline \multirow{4}{*}{$1: 25.000$} & A & $37 \%$ apresentaram elevação $\leq 2,7$ metros & \\
\hline & $\mathrm{B}$ & $53 \%$ apresentaram elevação $\leq 5$ metros & \multirow{11}{*}{8,35 metros } \\
\hline & $\mathrm{C}$ & $59 \%$ apresentaram elevação $\leq 6$ metros & \\
\hline & $\mathrm{D}$ & $67 \%$ apresentaram elevação $\leq 7,5$ metros & \\
\hline \multirow{4}{*}{$1: 50.000$} & A & $56 \%$ apresentaram elevação $\leq 5,5$ metros & \\
\hline & $\mathrm{B}$ & $79 \%$ apresentaram elevação $\leq 10$ metros & \\
\hline & $\mathrm{C}$ & $86 \%$ apresentaram elevação $\leq 12$ metros & \\
\hline & $\mathrm{D}$ & $93 \%$ apresentaram elevação $\leq 15$ metros & \\
\hline \multirow{4}{*}{$1: 100.000$} & A & $90 \%$ apresentaram elevação $\leq 13,7$ metros & \\
\hline & $\mathrm{B}$ & $99 \%$ apresentaram elevação $\leq 25$ metros & \\
\hline & $\mathrm{C}$ & $100 \%$ apresentaram elevação $\leq 30$ metros & \\
\hline & $\mathrm{D}$ & $100 \%$ apresentaram elevação $\leq 36,6$ metros & \\
\hline
\end{tabular}




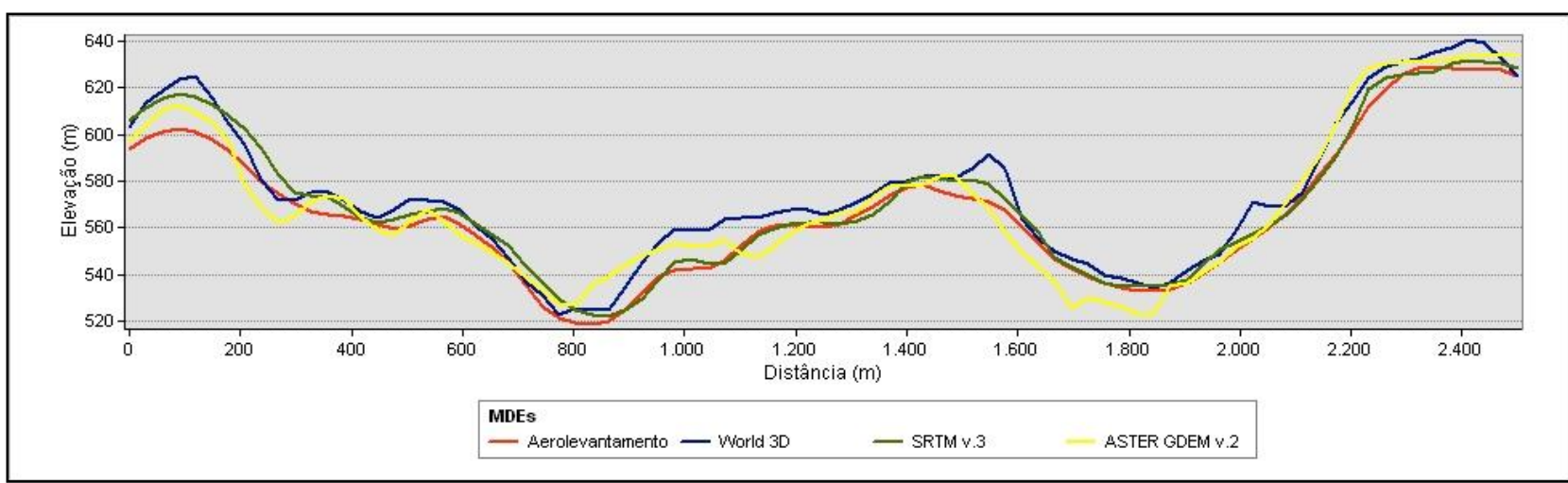

Figura 6. Perfil topográfico. Segmentos A/B dos perfis topográficos gerados a partir dos MDEs para a região da DO Vale dos Vinhedos.

No segmento C/D (Figura 7), o MDE SRTM v.3 foi o que apresentou maior semelhança com o MDE gerado pelo aerolevantamento, porém o modelo ASTER GDEM v.2 apresenta, da mesma forma, uma boa relação com o modelo gerado pelo aerolevantamento. Análises geomorfológicas, como entalhamento dos vales para verificação de rugosidade, podem ser influenciadas por estas diferenças de representação dos perfis topográficos.

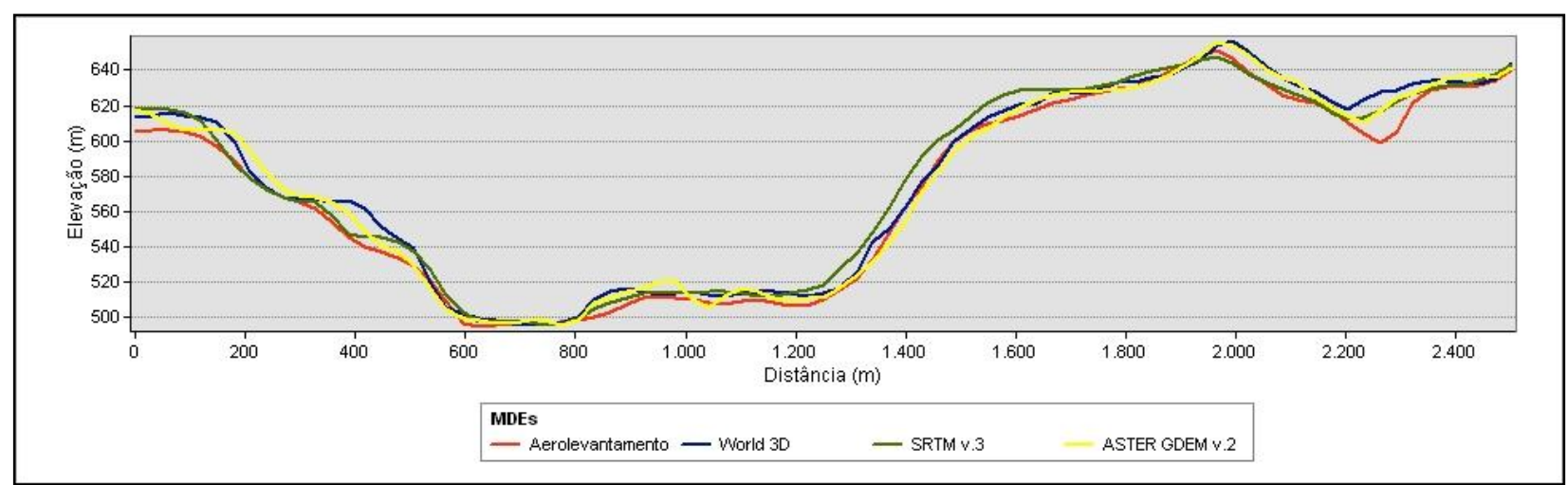

Figura 7. Perfil topográfico. Segmentos C/D dos perfis topográficos gerados a partir dos MDEs para a região da DO Vale dos Vinhedos.

Analisando os dois perfis, percebe-se que as principais diferenças entre os MDEs ocorreram nas regiões de fundo de vale. O modelo ALOS World 3D não apresentou boa relação morfológica nos dois perfis topográficos. Na Figura 8, pode-se observar a localização dos segmentos utilizados para gerar os perfis topográficos.

Viel, J. A., Rosa, K. K. Mendes Junior, C. W. 


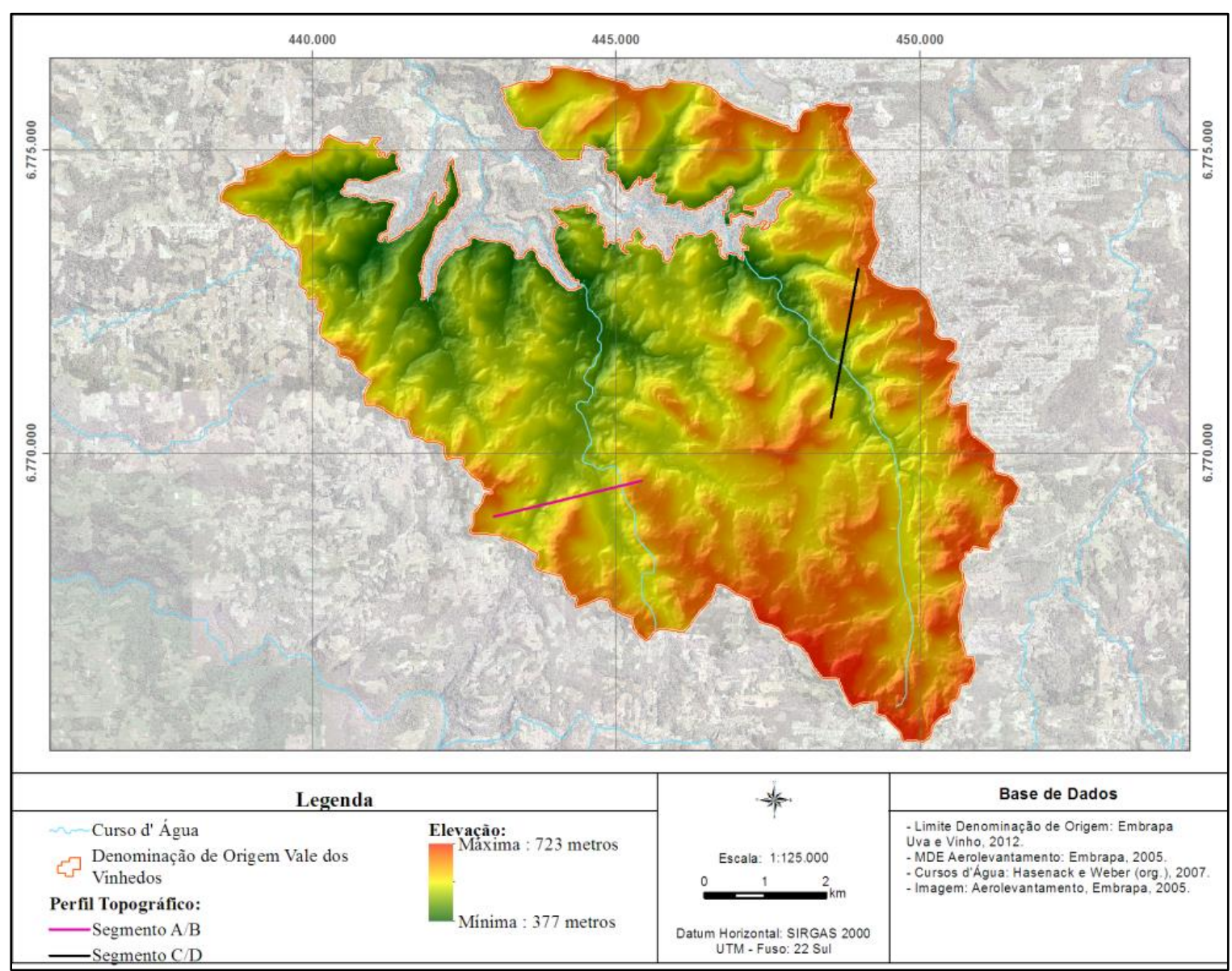

Figura 8. Mapa de localização dos segmentos A/B e C/D. Distribuição espacial dos segmentos gerados para a elaboração dos perfis topográficos.

\section{Conclusões}

As análises estatísticas e permitiram avaliar quantitativamente e qualitativamente os MDEs ALOS World 3D, SRTM v.3 e ASTER GDEM v.2. Esses modelos são utilizados para a realização de diversos estudos, tendo como ponto principal a sua gratuidade e a cobertura quase total da superfície terrestre.

Estatisticamente, o MDE que apresentou maior proximidade com o modelado da superfície terrestre foi o SRTM v.3, pois as elevações médias foram próximas às do MDE fotogramétrico. $\mathrm{Na}$ análise de correlação linear de Pearson esse modelo foi o que apresentou melhor resultado. Além disso, os dados de declividade derivados do MDE SRTM v.3 tiveram maior similaridade com os derivados do MDE fotogramétrico.

Quando analisado o PEC-PCD altimétrico, concluiu-se que os modelos podem ser utilizados para a escala 1:100.000, pois os mesmos enquadram-se nas classes A, B, C e D, com ressalvas para o modelo ALOS World 3D que enquadra-se somente a partir da classe B. Para a escala 1:50.000 os modelos foram compatíveis somente com a classe $\mathrm{D}$, enquanto que nenhum destes sequer chegou a essa classe na escala 1:25.000. Portanto, para a região da DO Vale dos Vinhedos recomenda-se não utilizar os MDEs analisados para estudos que necessitem escalas maiores do que 1:50.000.

Os perfis topográficos permitiram avaliar qualitativamente os MDEs ALOS World 3D, SRTM v.3 e ASTER GDEM v.2. Nesta análise, mais uma vez, o MDE SRTM v.3 apresentou maior relação com o MDE fotogramétrico. Os resultados podem estar relacionados ao método de aquisição dos MDEs analisados.

Por fim, cabe destacar que os modelos analisados apresentaram dados relativamente próximos, tanto estatisticamente, quanto morfologicamente. Sendo assim, para a utilização dos mesmos deve-se considerar o objetivo do estudo, a precisão desejada, bem como a escala de análise e de apresentação desses dados. 


\section{Agradecimentos}

Os autores agradecem à Universidade Federal do Rio Grande do Sul, à Embrapa Uva e Vinho e a CAPES pelo apoio prestado.

\section{Referências}

Araújo, P. V. do N., Amaro, V. E., Alcoforado, A.V. C., Santos A. L. S. dos., 2019. Acurácia Vertical e Calibração de Modelos Digitais de Elevação (MDEs) para a Bacia Hidrográfica Piranhas-Açú, Rio Grande do Norte, Brasil. Anuário do Instituto de Geociências - UFRJ, $351 \quad-\quad 364 . \quad$ DOI: http://dx.doi.org/10.11137/2018_1_351_364.

Brasil, 1984. Decreto Federal no 89.817, de 20 de junho.

CPRM - Serviço Geológico do Brasil. 2008. Mapa Geológico do Rio Grande do Sul: escala 1:750.000. Brasília.

Dias, F. de M., Miceli, B. S., Seabra, F. M., Santos, P. R. A. dos, Fernandes, M. do C, 2011. Avaliação Vertical de Modelos Digitais de Elevação (MDEs) em Diferentes Configurações Topográficas para Médias e Pequenas Escalas. Revista Brasileira de Cartografia, 63/1, 191201.

EB. Exército Brasileiro. Departamento de Ciência e Tecnologia. Diretoria de Serviço Geográfico, 2016. Especificação Técnica de Produtos de Conjuntos de Dados Geoespaciais, 2 ed. Brasília.

EB. Exército Brasileiro. Departamento de Ciência e Tecnologia. Diretoria de Serviço Geográfico. 2016. Norma da especificação técnica para aquisição de dados geoespaciais vetoriais de defesa da força terrestre, 2 ed. Brasília.

EMBRAPA. Empresa Brasileira de Pesquisa Agropecuária, 1979. Serviço Nacional de Levantamento e Conservação de Solos. Súmula da X Reunião Técnica de Levantamento de Solos. Rio de Janeiro.

Falcade, I., 2011. A Paisagem Como Representação Espacial: A Paisagem Vitícola Como Símbolo Das Indicações De Procedência De Vinhos Das Regiões Vale Dos Vinhedos, Pinto Bandeira e Monte Belo (Brasil). Porto Alegre. Tese (Doutorado). Porto Alegre, Universidade Federal do Rio Grande do Sul.

Feijoo, A. M. L. C. de, 2010. Correlação: A pesquisa e a estatística na psicologia e na educação. Rio de Janeiro, Centro Edelstein de Pesquisa Sociais, $70-77$.
Fran, L. L. S. D., Almeida, A. D. O. D., Penha, A. D. L. T. D., 2018. Avaliação da qualidade dos modelos digitais de elevação ASTER e SRTM para o estado da Bahia. Revista Brasileira de Cartografia, v. 69, nº 9, $1677-1686$.

Lousada, G., Fernandes, M. do C., 2017. Avaliação da precisão vertical de modelos digitais de elevação e análise de tendência em diferentes domínios geomorfológicos. Revista Brasileira de Cartografia. v. 69, $\mathrm{n}^{\circ}$ 7, 1263 - 1276.

Gaida, W., Breunig, F. M., Galvao, L. S., Teles, T. S., Balbinot, R., 2016. Variações da reflectância e dos índices de vegetação em função dos parâmetros da modelagem topográfica no Parque Estadual do Turvo, Rio Grande do Sul, Brasil. Boletín Del Instituto de Geografía, UNAM, 1-19. DOI: https://doi.org/10.14350/rig.52159.

Girão, R. S., Vicens, R. S., Fernandes, P. J. F., Barbalho, L. V., 2019. Avaliação da Acurácia Vertical de Modelos Digitais de Elevação (MDEs) para o Estado do Rio de Janeiro Através de Algoritmos de Automatização. Revista Caminhos de Geografia, 72 - 92.

Lang, H. R., Welch, R., 1999. Aster Digital Elevation Models - Algorithm Theoretical Basis Document. Disponível: https://eospso.nasa.gov/sites/default/files/atbd/ atbd-ast-14.pdf. Acesso: 19 Set. 2016.

Hoff, R., Ducati, J. R., Farias, A. R., Dalcin, M., 2015. Geologia, geomorfologia, sensoriamento remoto e SIG como suporte à caracterização da indicação geográfica Campanha para vinhos de qualidade, RS, Brasil. Feira de Santana, Universidade Estadual de Feira de Santana, 175-178.

Maune, D. F., Kopp, S. M., Crawford, C. A., Zervas, C. E., 2007. Introduction: Digital Elevation Models, Digital Elevation Model Technologies and Applications: The DEM Users Manual. 2. ed. American Society for Photogrammetry and Remote Sensing. Maryland, 43.

Mello, Y. R. de, Silveira, C. T. da, 2018. Análise de Modelos Digitais de Elevação e geração de atributos topográficos para a região da Serra do Mar do estado de Santa Catarina. Revista Brasileira de Geografia Física. V. 11, $n^{\circ} 06$, $2029-2045$.

MMA. Ministério do Meio Ambiente, 2004. Mapas de Cobertura Vegetal. Brasília.

Morais, J. D., Faria, T. S., Elmiro, M. A. T., Nero, M. A., Silva, A. de A., Nobrega, R. A. de A, 2017. Altimetry assessment of ASTER GDEM 
v. 2 and SRTM v.3 digital elevation models: a case study in urban area of Belo Horizonte, MG, Brazil. Boletim de Ciências Geodésicas, 23(4), 654-668.

DOI: http://dx.doi.org/10.1590/S1982217020170004 00043.

Nemirovsky, A. K. da S., Lousada, G., Antunes, F. de S., Fernandes, M. do C, 2017. Avaliação da acurácia vertical e horizontal de modelos digitais de elevação (SRTM PLUS, ALOS WORLD 3D E ASTER GDEM 2) a partir de um MDE de referência. Anais do XVIII Simpósio Brasileiro de Sensoriamento Remoto. Santos, INPE, 3561 -3568.

Robaina, L. E. DE S., Trentin, R., Bazzan, T., ReckziegeL, E. W., Verdum, R, 2010. Compartimentação Geomorfológica Da Bacia Hidrográfica Do Ibicuí, Rio Grande Do Sul, Brasil: Proposta De Classificação. Revista Brasileira de Geomorfologia, v. 11, n. 2, 11-23.

Rodriguez, E., Morris, C. S., Belz, J. E., Chapin, E., Martin, J., Hensley, S., 2006. An assessment of the SRTM topographic products. Photogrammetric Engineering and Remote Sensing, v. 72, n. 3, 249-260.

Santos, S. D. R., Huinca, S. C. M., Melo, L. F. D. S.; Silva, M. T. Q. S. Da, Delaza, L. S., 2010. Considerações sobre a utilização do PEC (Padrão de Exatidão Cartográfica) nos dias atuais. III Simpósio Brasileiro de Ciências Geodésicas e Tecnologias da Geoinformação, n. 2001, 1-5.

Silva, A. S. da, Santiago, O. R. P. L., Silva, C. R. da, 2018. Análise de Exatidão entre MDEs: AW3D, SRTM-30m e Projeto SPMGGO50. Geografia (Londrina) v. 27. $\mathrm{n}^{\circ} 2,87-98$. DOI: $10.5433 / 2447-1747.2018 v 27 \mathrm{n} 2 \mathrm{p} 87$

Souza, J. O. P. de, 2015. Análise da Precisão Altimétrica dos Modelos Digitais de Elevação para Área Semiárida do Nordeste Brasileiro. Revista do Departamento de Geografia -USP, v. 30, 56-64.
Steinke, V. A., Sano, E. E, 2011. Semi-Automatic Identification Gis-Based Morphometry of Geomorphic Features of Federal District of Brazil. Revista Brasileira de Geomorfologia, v. 1, 3-9.

Tachikawa, T., Kaku, M., Iwasaki, A., Gesch, D., Oimoen, M., Zhang, Z., Danielson, J., Krieger, T., Curtis, B., Haase, J., Abrams, M., Crippen, R., Carabajal, C, 2011. ASTER Global Digital Elevation Model Version 2 - Summary of Validation Results. Japan Space Systems, 27. Disponível:http://www.jspacesystems.or.jp/ers dac/GDEM/ver2Validation/Summary_GDEM2 _validation_report_final.pdf. Acesso: 25 jul. 2017.

Tadono, T., Ishida, H., Oda, F., Naito, S., Minakawa, K., Iwamoto, H, 2014. Precise Global DEM Generation by ALOS PRISM. ISPRS Annals of Photogrammetry, Remote Sensing and Spatial Information Sciences, v. II4, 71-76. Disponível: http://www.isprs-annphotogramm-remote-sens-spatial-inf-sci.net/II4/71/2014/. Acesso: $10 \mathrm{dez} .2017$.

Tonietto, J., Zanus, M. C., Falcade, I., Guerra, C. C., 2012. Regulamento de Uso da Denominação de Origem Vale dos Vinhedos Vinhos Finos Tranquilos e Espumantes. Bento Gonçalves, 35.

Valeriano, M. D. M., 2008. Topodata: Guia Para Utilização De Dados. São José dos Campos, INPE, 73.

Viel, J. A., Rosa, K. K. Da, Hoff, R., 2017. Estudo da erosão superficial do solo por meio de SIG na região da Denominação de Origem Vale dos Vinhedos (Brasil). Revista Brasileira de Geomorfologia, 521 - 533.

Viel, J. A., Rosa, K. K. Da, Hoff, R., 2017. Avaliação do Efeito do Dossel Vegetativo na Estimativa da Perda do Solo em Área de Vinhedos Usando SIG. Revista Brasileira de Cartografia, $n^{\circ}$ 69/6, $1019-1028$. 